\title{
BMJ Open Factors that facilitate or hinder whole system integrated care for obesity and mental health: a scoping review protocol
}

\author{
Fani Liapi (D) , ${ }^{1}$ Angel Chater, ${ }^{2}$ Gurch Randhawa (D) , ${ }^{1}$ Yannis Pappas (D) ${ }^{1}$
}

To cite: Liapi F, Chater A, Randhawa $\mathrm{G}$, et al. Factors that facilitate or hinder whole system integrated care for obesity and mental health: a scoping review protocol. BMJ Open 2021;11:e050527. doi:10.1136/ bmjopen-2021-050527

- Prepublication history and additional supplemental material for this paper are available online. To view these files, please visit the journal online. (http://dx.doi.org/10.1136/ bmjopen-2021-050527).

Received 02 March 2021 Accepted 02 July 2021

\section{ABSTRACT}

Introduction Integrated care aims to improve population health. Obesity and mental health are major health issues worldwide. The complexity of the multifactorial drivers of these public health problems has led to the adoption of a whole system approach. This review aims to highlight factors that influence the planning, implementation and evaluation of whole system integrated care for these conditions.

Methods and analysis Using the framework of Arksey and 0 ' Malley, we will perform a comprehensive search in the following databases: MEDLINE, CINAHL, PsychINFO, PubMed, British Nursing Database, Web of Science, Health Systems Evidence, Cochrane Library and University of York Centre for Reviews and Dissemination. Further hand-search of reference lists and the grey literature will be conducted. The search will be restricted to articles published from 2000 to 2020 . The review is expected to be completed by August 2021. Full texts of the potential studies will be screened for the inclusion criteria. Quality of studies will be appraised. Narrative synthesis will be completed using data extracted from the included studies. Ethics and dissemination A favourable ethics opinion for this study was obtained from the Institute for Health Research Ethics Committee of the University of Bedfordshire (IHREC937). This review expects to identify information relating to factors that facilitate or hinder whole system integrated care for obesity and mental health. The finding from this review will be widely disseminated to stakeholders to inform implementation of whole system integrated care initiatives.

\section{BACKGROUND}

The National Health System (NHS) 5-Year Forward View Plan, that was published in 2014, outlined a transformation of services to enable a sustainable health and social care system. ${ }^{1}$ The recently published 'NHS Long-Term Plan' recognises that the NHS still faces some key challenges such as funding, increasing inequalities and pressures from a growing and ageing population. ${ }^{2}$ Integrated care is perceived as a means to address the challenges that the health and social care systems are facing. ${ }^{3} 4$ The recommendations to use the principles of integrated care to meet

\section{Strengths and limitations of this study}

This is the first scoping review to provide a comprehensive synthesis of factors that facilitate or hinder the implementation of integrated interventions for obesity and mental health, under the whole system approach.

- Using narrative synthesis is one of the strengths of this scoping review as it allows to combine quantitative, qualitative and mixed methods studies.

- Quality appraisal of included studies will be completed.

- The review will be restricted to articles published in English and this may serve as a drawback to this study.

the complex needs of health and social care users derive from the view that integrated care could provide a platform that enables co-ordinated and patient-centred care across several sectors and agencies. ${ }^{3}$ Taking into consideration the perceived benefits of integrated care, policy-makers aim to develop and test new models of integrated care that will improve the quality of healthcare without placing extra pressure on the available resources. ${ }^{5}$ Some of the attempted approaches, such as place-based and community-centred approach, move beyond the integration of organisations as they aim to reach a 'whole system' integration across healthcare sectors and the community. ${ }^{78}$

The term 'whole system approach' indicates that there is no one key answer to resolve complex public health issues and responds to complexity through a flexible way of working. ${ }^{9}{ }^{10}$ A local whole system approach enables stakeholders, including communities, commissioners and healthcare providers, to connect and share an understanding of the challenge. Stakeholders work together in a joined-up way to identify the most possible opportunities for change and to decide ways of action. ${ }^{10}$ 
In 2019, Public Health England published a guide to support local authorities with the implementation of interventions to tackle obesity, based on the whole system approach. ${ }^{11}$ Recently, another report has been published from Public Health England, which highlights the key elements that are required to make a shift to whole system approaches in community-centred public health. ${ }^{12}$

Obesity is a major health issue worldwide. Specifically, in the UK, the prevalence of obesity has more than doubled in the last 25 years. ${ }^{13}$ The Foresight report (2007) highlights the social and biological complexity in managing obesity. The obesity system map is created by a large number of variables such as cultural, environmental and psychological. ${ }^{13}$ Therefore, there is no one solution to tackle a complex multifaceted system of causes. In 2019, Public Health England published a guide to support local authorities to create their whole systems approaches to tackle obesity. ${ }^{11}$ A recent systematic review ${ }^{14}$ provides evidence for the effectiveness of system approaches to tackle obesity and highlights the lack of evidence of how to operationalise a whole system approach to address complex public health issues. This scoping review aims to fill this gap and provide evidence of the factors that facilitate or hinder the planning, implementation and evaluation of whole system integrated care interventions targeting complex public health issues, such as obesity and mental health.

There is a strong evidence in the literature that obesity is associated with several mental health conditions, such as depression and anxiety disorders. ${ }^{15-20}$ The rising prevalence of obesity and mental health conditions represents two important public health issues. A meta-analysis conducted by Pereira-Miranda et $a t^{20}$ shows that the probability of developing depression was $32 \%$ higher $(\mathrm{OR}=1.32$; 95\% CI 1.26 to 1.38 ) among individuals living with obesity compared with individuals in healthy weight. Another meta-analysis shows a significantly higher frequency of anxiety in obesity $(\mathrm{OR}=1.30 ; 95 \%$ CI 1.20 to $1.41, \mathrm{p}<0.001)$ and those classified as overweight $(\mathrm{OR}=1.10 ; 95 \%$ CI 1.00 to $1.21, \mathrm{p}=0.049) .{ }^{18}$ Therefore, it is not a surprise that the Public Health England and NHS are focusing on ways of managing effectively these growing complex public health issues and recognise the need of more co-ordinated health and social care. ${ }^{21}$ The 5 -Year Forward View for Mental Health highlights the need for the development of more integrated approaches to mental health. ${ }^{22}$ In answer to this, NHS England announced the plans to invest in the development of integrated support focusing on physical and mental health needs across the whole system. ${ }^{23}$

To our knowledge, no existing review outlines the factors that facilitate or hinder the planning, implementation and evaluation of whole system integrated care intervention for obesity and mental health. The aim of this scoping review is to fill this gap, by collecting and synthesising evidence on the factors that facilitate and hinder the planning, implementation and evaluation of whole system integrated interventions for obesity and mental health and the impact on those interventions on patients' physiological (body mass index (BMI)) and psychological (depression and anxiety) outcomes.

This scoping review will be carried out as part of a wider study that aims to understand the impact of the whole system approach to integrated care interventions. The expected impact of the scoping review is to inform health system improvements by collecting and synthesising key implementation components of integrated care.

\section{METHODS AND ANALYSIS}

This scoping review will follow the framework presented by Arksey and O' Malleys ${ }^{24}$ that entails the following six steps: identifying the research question, searching for relevant studies, selecting studies, charting the data, collating, summarising and reporting the results and conducting consultation exercises. The last step is optional, and it will not be included in this scoping review. Part of the process of systematically assessing the outcome of scientific evidence is to critically appraise research studies. ${ }^{25}$ Therefore, this scoping study will appraise the quality of evidence in the included studies.

As 'comprehensiveness is the whole point of scoping a field', ${ }^{24}$ a broad search strategy will be employed. The development of the search strategy was guided by the Population-Intervention-Comparison-Outcome) framework which defines the above elements of interest. The following table (table 1) demonstrates the process that we used to formulate the research questions.

To guide the research process, the following definitions were also developed:

- 'Integrated care' refers to organisational models designed to provide proactive, person-centred, holistic, coordinated and multidisciplinary care by two or more collaborating providers. Providers may work at the same or different organisation within the healthcare sector, including health, social and community care sectors. Full integration has been identified as the third level of integration where pool funding exists, responsibilities and resources are collaboratively organised and structured to define new benefits and services. Full integration is required for users with high levels of need, with complex problems including behaviour problems. ${ }^{26-28}$

- 'Whole systems approach' enables stakeholders, including communities, to connect, share an understanding of the challenge, consider how the system is operating, and identify potential opportunities for change. A whole systems approach does not offer a single technique rather it provides a framework within which most or all of the participants can agree to an agenda for improvement or a process for moving forward. ${ }^{29}$

- 'Model' refers to framework or theory for integrated care. 


\begin{tabular}{ll}
\hline Table 1 PICOs criteria in framing the research question \\
\hline Population & Any population, adults or children, \\
including any sex and age, globally
\end{tabular}

BMI, body mass index; PICOs, Population-InterventionComparison-Outcomes.

- 'Intervention' refers to organised effort that attempts to improve mental and physical health on a population level.

\section{Stage 1: identifying the research questions}

The primary aim of this review is to summarise the existing evidence of barriers and facilitators of successful planning, implementation and evaluation of whole systems integrated care for obesity and mental health. The secondary aim is to understand the impact of whole systems integrated care on patients' physiological (BMI) and psychological (depression and anxiety) effects.

The broad question that guides this review is "What is the existing evidence of whole systems integrated care interventions for obesity and mental health?' To create a distinct purpose of this review and facilitate the next steps, the following research questions will guide this review:

1. What are the factors that facilitate and hinder the implementation of whole systems integrated interventions for obesity and mental health?

2. What is the impact of whole systems integrated interventions on the patients' physiological (BMI) and psychological (depression and anxiety) outcomes?

\section{Stage 2: identifying relevant studies}

This review aims to summarise the factors that facilitate or hinder the planning, implementation and evaluation of whole systems integrated care interventions for obesity and mental health and understand the impact of those interventions. Therefore, qualitative, quantitative and mixed methods studies will be included in the scoping review. Evaluation reports are also included in the review.

At this phase, relevant studies will be identified and search strategy, terms/concepts to use, sources to be searched, language will be developed. As 'comprehensiveness is the whole point of scoping a field', ${ }^{24}$ a broad search strategy will be employed. The search will be restricted to articles published from 2000 to 2020. The review is on-going and is expected to be completed by August 2021. Research literature will be identified via electronic searches in the following databases: MEDLINE, The Cumulative Index to Nursing and Allied Health Literature (CINAHL), PsycINFO, PubMed, British Nursing Database and Web of Science. To identify relevant interventions in the UK, the websites of the following organisation will be searched: The Kings Fund, Nuffield Trust, Department of Health, National Institute for Health and Care Excellence (NICE) and The Health Foundation. Additionally, a wide handsearching and grey literature search will be conducted in Open-Grey, Google Scholars and Google, to identify any unpublished studies, fact sheets, government documents, white papers, relevant to this scoping review. In addition, the reference lists of all the relevant studies and reports will be searched.

The databases will be searched for English language studies using the search terms specified. The following search strategy/keywords will be used to identify relevant studies: 'integrated care' OR 'multidisciplinary care' OR 'holistic care' OR 'joint care' OR 'coordinated care' or 'person-centred care' OR integrat* OR 'whole system' OR 'whole system approach' AND 'weight management' OR obesity OR overweight OR 'body mass index' OR BMI OR 'weight gain' OR 'mental health service*' OR anxiety OR depression. A draft of the search strategy for Medline can be found in the online supplemental material. The search strategy has been discussed and agreed with co-authors and the Academic Liaison Librarian for healthcare practice of the University of Bedfordshire.

\section{Stage 3: selection of studies}

To increase the rigour of the review, the research team discussed and determined the inclusion and exclusion criteria before the research begins. ${ }^{30}$

\section{Inclusion criteria}

- A model of whole systems integrated care is described and/or evaluated (evaluation of implementation processes, not intervention outcomes). The model has to be aligned with the above-described definition of whole systems approach.

- Key elements of whole systems integrated care are described. 
- The focus of the article is on the development of a whole systems integrated care intervention in any location of care (hospital and community).

- The intervention is focused on any target population, including people aged $<18$ years, pregnant women, older people.

- Only studies written in English will be included.

- As a scoping review aims to cover a wide range of literature, there will be no restrictions of the type of studies that will be included in this review.

\section{Exclusion criteria}

- No full-text paper was available.

- They were not written in English language.

A two-stage selection process, which involves screening of titles and abstracts, followed by full-text screening, will be used to determine eligible studies. The extracted data will be represented using evidence mapping and a narrative summary.

After eliminating the duplicates, an initial screening of titles and abstracts will be conducted, by one author (FL), to exclude records that do not meet the inclusion criteria. Where there is ambiguity, input will be sought from a second reviewer (YP). Subsequently, a second review author (YP) will conduct a check on a random sample $10 \%$ of the screened articles. The full-text screening phase includes all the records that will meet the inclusion criteria based on the title and abstract only. Agreement between the reviewers will be assessed using the percent agreement statistic, to determine whether further reviewing is required. If the interrater reliability is $<82 \%$, almost perfect agreement, an additional subset of citations $(25 \%)$ will be doubled screened. Any disagreement between reviewers will be resolved through discussion between the first and second reviewers or by the research team, when consensus cannot be reached. Reference lists of included articles will also be reviewed for potential eligible studies. An adapted Preferred Reporting Items for Systematic Reviews and Meta-Analyses flow-chart of study selection will be completed and included in the review.

\section{Stage 4: data extraction}

The extraction process for a scoping review is known as 'charting data' and presents information about the study that aligns with the objectives and research questions of the review. ${ }^{24}$ Suitable data from the eligible studies will be extracted manually using a customised Microsoft Excel sheet. The following extracted data will be included in the review: authors, year, study design, country, description of intervention, identified enablers, identified barriers and outcomes (see box 1). The development of this extract sheet will facilitate the identification of factors/processes that are reported to impact the implementation of an intervention and describes patterns across the studies in terms of the direction of effects. ${ }^{31}$

\section{Stage 5: summarising and reporting the results}

Narrative synthesis has been described as the 'second best' approach to synthesise findings from multiple studies,

\section{Box 1 Data extraction form}

Authors and year.
Study design.
Country.
Description of intervention.
Identified enablers.
Identified barriers.
Outcomes.

when data cannot be analysed using meta-analysis. ${ }^{31} 32$ However, narrative synthesis is not simply describing and summarising the qualitative findings of each study, but can be used to synthesise both quantitative and qualitative studies. It has also been used when experimental and quasi-experimental studies are included in a review and due to heterogeneity of the findings, meta-analysis cannot be conducted. ${ }^{33}$ It is expected that terminology of barriers and facilitators and metrics will vary between studies. Therefore, a narrative approach will be needed. The narrative synthesis in this study will be conducted based on the guidelines by Popay et $a l^{31}$ and Ryan and The Cochrane Consumers and Communication Review Group. $^{32}$. Narrative synthesis helps explain and interpret results of synthesis of evidence on 'what works', which can be useful in informing policy and practice.

\section{Stage 6: assessment of methodological quality}

This study follows the methodological framework proposed by Arksey and O'Malley. ${ }^{24}$ Although Arksey and O'Malley argued that 'quality assessment does not form part of the scoping study remit', they appreciated that this may be a limitation of the method. ${ }^{24}$ Their main argument in terms of the lack of quality assessment is that it reduces the quantity of studies included in the review. ${ }^{24}$ This impede the main aim of a scoping review; to capture a broad range of evidence of a field. This may justify the lack of quality assessment on scoping reviews and the acknowledgement that is a limitation of the studies. ${ }^{34}$ Grant and Booth (2009) stated that the lack of quality assessment in scoping reviews does not encourage the use of their finding to inform police/practice. It is suggested that the development of a critical appraisal tool for scoping study quality should be considered. ${ }^{34}{ }^{35}$ The purpose of this scoping review is to inform health system improvements by collecting and synthesising key implementation components of integrated care. Therefore, quality appraisal is critical in ensuring studies' trustworthiness, value and relevance in evidence-based practice and policy. The quality assessment scores will be included in a table to show the strength of the evidence for each study.

of quality assessment in scoping reviews does not encourage the use of their finding to inform police/ practice. It is suggested that the development of a critical appraisal tool for scoping study quality should be considered. ${ }^{34}{ }^{35}$ The purpose of this scoping review is to inform 
health system improvements by collecting and synthesising key implementation components of integrated care. Therefore, quality appraisal is critical in ensuring studies' trustworthiness, value and relevance in evidencebased practice and policy. The quality assessment scores will be included in a table to show the strength of the evidence for each study.

\section{DISCUSSION}

This scoping review aims to summarise the enablers and inhibitors of implementing a whole system integrated intervention to tackle obesity and improve mental health and to obtain an insight into the impact of whole system interventions on resource, economic and health outcomes. This study will provide a structured framework based on the synthesis of the literature that might be used towards enhancing whole system approaches to complex health problems such as obesity and mental health. The narratively synthesised findings of this review can inform policymakers and relevant stakeholders in their initiatives to implement a whole system integrated intervention to tackle obesity and improve mental health.

\section{Limitations}

There are limitations of this review that warrant consideration. The review will be limited to English publications only, because of limited resources for language translation. Also, a single-reviewer data extraction (potential bias) is consider an important limitation of a review. In addition, patient and public are not involved in this study. Although, the study involves stakeholders involvement in different phases.

\section{Patient and public involvement}

Patients and the public were not involved in the development of this protocol.

\section{ETHICS AND DISSEMINATION}

This is a scoping review that will only use secondary data from existing studies, which have granted ethics approval. The study is approved by the Institute for Health Research Ethics Committee (IHREC) of the University of Bedfordshire, UK (IHREC937).

The findings of this review will be shared through a peer-reviewed journal publication and will be disseminated with stakeholders and commissioners involved in the implementation of integrated care initiatives under a whole system approach. Also, the review findings will inform a multistage evaluation study.

Acknowledgements The authors thank David Abdy, the academic liaison librarian for healthcare practice of the University of Bedfordshire, for his guidance with the development of search strategy.

Contributors FL drafted the protocol. The protocol was reviewed by YP, AC and GR, who provided critical insights and recommendations as well as edits. All authors have approved and contributed to the final manuscript.
Funding The study is part of a PhD studentship jointly funded by the Luton Clinical Commissioning Group, Turning Point and University of Bedfordshire.

Competing interests None declared.

Patient consent for publication Not required.

Provenance and peer review Not commissioned; externally peer reviewed.

Supplemental material This content has been supplied by the author(s). It has not been vetted by BMJ Publishing Group Limited (BMJ) and may not have been peer-reviewed. Any opinions or recommendations discussed are solely those of the author(s) and are not endorsed by BMJ. BMJ disclaims all liability and responsibility arising from any reliance placed on the content. Where the content includes any translated material, BMJ does not warrant the accuracy and reliability of the translations (including but not limited to local regulations, clinical guidelines, terminology, drug names and drug dosages), and is not responsible for any error and/or omissions arising from translation and adaptation or otherwise.

Open access This is an open access article distributed in accordance with the Creative Commons Attribution Non Commercial (CC BY-NC 4.0) license, which permits others to distribute, remix, adapt, build upon this work non-commercially, and license their derivative works on different terms, provided the original work is properly cited, appropriate credit is given, any changes made indicated, and the use is non-commercial. See: http://creativecommons.org/licenses/by-nc/4.0/.

\section{ORCID iDs}

Fani Liapi http://orcid.org/0000-0002-4861-1527

Gurch Randhawa http://orcid.org/0000-0002-2289-5859

Yannis Pappas http://orcid.org/0000-0003-3087-860X

\section{REFERENCES}

1 NHS England. NHS five year forward view plan, 2014.

2 NHS England. The NHS long term plan. NHS England, 2019.

3 Goddard M, Mason AR. Integrated care: a pill for all ills? Int $J$ Health Policy Manag 2017;6:1-3.

4 Kodner DL. All together now: a conceptual exploration of integrated care. Healthc Q 2009;13:6-15 http://www.ncbi.nlm.nih.gov/pubmed/ 20057243

5 NHS England. People helping people. year two of the pioneer programme. London: NHS England, 2016.

6 NHS England. New care model: vanguards - developing a blueprint for the future of NHS and care services [online]. London: NHS England, 2016. https://www.england.nhs.uk/wp-content/uploads/ 2015/11/new_care_models.pdf

7 Ham C, Alderwick H. Place-based health systems [online]. The King's Fund, 2015. http://www.kingsfund.org.uk/sites/files/kf/field/field_ publication_file/Place-based-systems-of-care-Kings-Fund-Nov2015_0.pdf

8 Public Health England. A guide to community-centred approaches for health and wellbeing full report, 2015.

9 Rutter H, Savona N, Glonti K, et al. The need for a complex systems model of evidence for public health. Lancet 2017;390:2602-4.

10 Public Health England. Whole systems approach to obesity programme learning from co-producing and testing the guide and resources about public health England, 2019.

11 Public Health England. Whole systems approach to obesity: a guide to support local approaches to promoting a healthy weight, 2019.

12 Public Health England. Community-centred public health taking a whole system approach about public health England, 2020.

13 Government Office for Science. Tackling obesities: future choices project report. London, 2007.

14 Bagnall A-M, Radley D, Jones R, et al. Whole systems approaches to obesity and other complex public health challenges: a systematic review. BMC Public Health 2019;19:1-14.

15 de Wit L, Luppino F, van Straten A, et al. Depression and obesity: a meta-analysis of community-based studies. Psychiatry Res 2010;178:230-5.

16 Roberts RE, Deleger S, Strawbridge WJ, et al. Prospective association between obesity and depression: evidence from the Alameda County study. Int J Obes Relat Metab Disord 2003;27:514-21.

17 Gariepy G, Nitka D, Schmitz N. The association between obesity and anxiety disorders in the population: a systematic review and metaanalysis. Int J Obes 2010;34:407-19.

18 Amiri S, Behnezhad S. Obesity and anxiety symptoms: a systematic review and meta-analysis. Neuropsychiatr 2019;33:72-89. 
19 Moussa OM, Ardissino M, Kulatilake P, et al. Effect of body mass index on depression in a UK cohort of 363037 obese patients: a longitudinal analysis of transition. Clin Obes 2019;9:e12305.

20 Pereira-Miranda E, Costa PRF, Queiroz VAO, et al. Overweight and obesity associated with higher depression prevalence in adults: a systematic review and meta-analysis. J Am Coll Nutr 2017;36:223-33.

21 Public Health England. PHE strategy 2020-25, 2019.

22 NHS England. Five year forward view for mental health: one year on [online]. NHS England, 2017. https://www.england.nhs.uk/wpcontent/uploads/2017/03/fyfv-mh-one-year-on.pdf

23 Naylor C, Taggart H, Charles A. New models of care in mental health NHS [online], 2017. Available: https://www.kingsfund.org.uk/sites/ files/kf/field/field_publication_file/MH_new_models_care_Kings_ Fund May 2017.pdf

24 Arksey H, O'Malley L. Scoping studies: towards a methodological framework. Int J Soc Res Methodol 2005;8:19-32.

25 Mhaskar R, Emmanuel P, Mishra S, et al. Critical appraisal skills are essential to informed decision-making. Indian J Sex Transm Dis AIDS 2009;30:112.

26 Nies H. Managing effective partnerships in older people's services. Health Soc Care Community 2006;14:391-9.

27 Rand Europe and Ernst \& Young. National evaluation of the department of health's integrated care pilots. Final report: full version [online]. Department of Health, 2012. Available: https://www.gov.uk/ government/uploads/system/uploads/attachment_data/file/215103/ dh_133127.pdf

28 Leutz WN. Five laws for integrating medical and social services: lessons from the United States and the United Kingdom. Milbank Q 1999;77:77-110.

29 Hudson B. Whole systems working: a guide and discussion paper, 2006.

30 Levac D, Colquhoun H, O'Brien KK. Scoping studies: advancing the methodology. Implement Sci 2010;5.

31 Popay J, Roberts H, Sowden A, et al. Guidance on the conduct of narrative synthesis in systematic reviews: a product from the ESRC methods programme, 2006.

32 Ryan, Cochrane Consumers and Communication Review Group. Cochrane Consumers and Communication Review Group: data synthesis and analysis, 2013.

33 Mays N, Pope C, Popay J. Systematically reviewing qualitative and quantitative evidence to inform management and policy-making in the health field. J Health Serv Res Policy 2005;10:6-20.

34 Pham MT, Rajić A, Greig JD, et al. A scoping review of scoping reviews: advancing the approach and enhancing the consistency. Res Synth Methods 2014;5:371-85.

35 Grant MJ, Booth A. A typology of reviews: an analysis of 14 review types and associated methodologies. Health Info Libr J 2009;26:91-108. 